\title{
SMALL-ENERGY ASYMPTOTICS FOR THE SCHRÖDINGER EQUATION ON THE LINE
}

Dedicated to Pierre C. Sabatier on the occasion of his 65th birthday

\author{
Tuncay Aktosun \\ Department of Mathematics \\ North Dakota State University \\ Fargo, ND 58105, USA \\ and \\ Martin Klaus \\ Department of Mathematics \\ Virginia Polytechnic Institute and State University \\ Blacksburg, VA 24061, USA
}

\begin{abstract}
The one-dimensional Schrödinger equation is considered when the potential is real valued, integrable, and has a finite first moment. The small-energy asymptotics of the logarithmic spatial derivative of the Jost solutions are established. Some consequences of these asymptotics are presented such as the small-energy limits of the scattering coefficients and a simplified characterization of the scattering data for the inverse scattering problem. When the potential also has a finite second moment, some improved results are given on the small-energy asymptotics of the scattering coefficients and the logarithmic spatial derivatives of the Jost solutions.
\end{abstract}

PACS Numbers (1999): 02.60.Lj, 03.65.Nk

Mathematics Subject Classification (2000): 34L25, 34L40, 81U05, 81U40

Keywords: 1-D Schrödinger equation, Small-energy asymptotics of Jost solutions, Smallenergy asymptotics of scattering coefficients, Riccati equation, Inverse scattering problem, Characterization of scattering data

Short title: Small-energy asymptotics of the Jost solutions 


\section{INTRODUCTION}

Consider the one-dimensional Schrödinger equation

$$
\psi^{\prime \prime}(k, x)+k^{2} \psi(k, x)=V(x) \psi(k, x), \quad x \in \mathbf{R},
$$

where the potential $V$ is real valued and belongs to $L_{1}^{1}(\mathbf{R})$. Here the prime denotes the derivative with respect to the spatial variable $x$, and $L_{n}^{1}(\mathbf{R})$ denotes the potential class in which $\int_{-\infty}^{\infty} d x(1+|x|)^{n}|V(x)|$ is finite.

The Jost solution from the left, $f_{l}(k, x)$, associated with $V$ is the solution of (1.1) satisfying

$$
e^{-i k x} f_{l}(k, x)=1+o(1), \quad e^{-i k x} f_{l}^{\prime}(k, x)=i k+o(1), \quad x \rightarrow+\infty .
$$

Thus, it can be obtained from the integral equation

$$
f_{l}(k, x)=e^{i k x}+\frac{1}{k} \int_{x}^{\infty} d y \sin k(y-x) V(y) f_{l}(k, y) .
$$

Similarly, $f_{r}(k, x)$, the Jost solution from the right, is the solution of (1.1) satisfying

$$
e^{i k x} f_{r}(k, x)=1+o(1), \quad e^{i k x} f_{r}^{\prime}(k, x)=-i k+o(1), \quad x \rightarrow-\infty .
$$

For each fixed $x \in \mathbf{R}$, the Jost solutions and their $x$-derivatives are analytic in $k \in \mathbf{C}^{+}$and continuous in $\overline{\mathbf{C}^{+}}$. We use $\mathbf{C}^{+}$to denote the upper-half complex plane and $\overline{\mathbf{C}^{+}}:=\mathbf{C}^{+} \cup \mathbf{R}$.

The transmission coefficient $T$, the reflection coefficient from the left $L$, and the reflection coefficient from the right $R$ are obtained from the spatial asymptotics

$$
\begin{aligned}
& e^{-i k x} f_{l}(k, x)=\frac{1}{T(k)}+\frac{L(k)}{T(k)} e^{-2 i k x}+o(1), \quad x \rightarrow-\infty, \\
& e^{i k x} f_{r}(k, x)=\frac{1}{T(k)}+\frac{R(k)}{T(k)} e^{2 i k x}+o(1), \quad x \rightarrow+\infty .
\end{aligned}
$$

Alternatively, these scattering coefficients can be obtained from

$$
\frac{2 i k}{T(k)}=\left[f_{r}(k, x) ; f_{l}(k, x)\right], \quad k \in \overline{\mathbf{C}^{+}},
$$




$$
\begin{aligned}
& \frac{2 i k R(k)}{T(k)}=\left[f_{l}(-k, x) ; f_{r}(k, x)\right], \quad k \in \mathbf{R}, \\
& \frac{2 i k L(k)}{T(k)}=\left[f_{l}(k, x) ; f_{r}(-k, x)\right], \quad k \in \mathbf{R},
\end{aligned}
$$

where $[f ; g]:=f g^{\prime}-f^{\prime} g$ denotes the Wronskian. It is known that $|L(k)|<1$ and $|R(k)|<1$ for $k \in \mathbf{R} \backslash\{0\}$, and hence none of the four functions $f_{l}(k, \cdot), f_{r}(k, \cdot), f_{l}^{\prime}(k, \cdot)$, and $f_{r}^{\prime}(k, \cdot)$ can vanish when $x \in \mathbf{R}$ for $k \in \mathbf{R} \backslash\{0\}$. We refer the reader to [Fa64,DT79,CS89] for the basic facts on the scattering theory for (1.1).

The potential $V$ is 'generic' if $f_{l}(0, x)$ and $f_{r}(0, x)$ are linearly independent, and it is 'exceptional' if $f_{l}(0, x)$ and $f_{r}(0, x)$ are linearly dependent. In the exceptional case we have

$$
\gamma=\frac{f_{l}(0, x)}{f_{r}(0, x)}, \quad x \in \mathbf{R}
$$

for some real, nonzero constant $\gamma$.

The behavior of the scattering coefficients at $k=0$ is related to the behavior of the potential as $x \rightarrow \pm \infty$. As we discuss in Section 5 , these behaviors play a crucial role in the solution of the inverse scattering problem for (1.1). On the other hand, determining the behavior of the scattering coefficients at $k=0$, especially in the exceptional case, requires elaborate and lengthy estimates if one assumes only $V \in L_{1}^{1}(\mathbf{R})$. In this paper we establish the small- $k$ asymptotics of the logarithmic spatial derivatives of the Jost solutions, from which the behavior at $k=0$ of the scattering coefficients is easily obtained.

This paper is organized as follows. Section 2 deals with the small- $k$ asymptotics of the Jost solutions. Our main result is given in Theorem 2.3, where by assuming only $V \in L_{1}^{1}(\mathbf{R})$, we prove that the logarithmic derivatives $f_{l}^{\prime}(\cdot, x) / f_{l}(\cdot, x)$ and $f_{r}^{\prime}(\cdot, x) / f_{r}(\cdot, x)$ can be differentiated with respect to $k$ at $k=0$. In Section 3 we discuss two consequences of Theorem 2.3; namely, we show that the small- $k$ asymptotics of the scattering coefficients 
in the exceptional case readily follow from Theorem 2.3 and that the result of this theorem is closely related to the recent result contained in Theorem 8.1 and Corollary 8.2 of [Ak99] on the small- $k$ asymptotics of the reflection coefficients associated with potentials whose supports are confined to a half line. In Section 4 we consider the small- $k$ asymptotics when the potential $V$ belongs to $L_{2}^{1}(\mathbf{R})$, and we improve the asymptotic results and present some consequences. In Section 5 we provide a reformulation of necessary and sufficient conditions on a scattering data so that it corresponds to a unique real-valued potential in $L_{1}^{1}(\mathbf{R})$. Finally, in the Appendix we give an independent proof of Theorem 2.3 by showing that certain solutions of the Riccati equation (A.1) are differentiable in $k$ at $k=0$. Note that all the results in Sections 2, 3, and 5 and the Appendix hold by assuming only $V \in L_{1}^{1}(\mathbf{R})$, and the stronger assumption $V \in L_{2}^{1}(\mathbf{R})$ is used only in Section 4 .

When $V \in L_{1}^{1}(\mathbf{R})$, for each $x \in \mathbf{R}$ we can in general only conclude that

$$
\begin{array}{ll}
f_{l}(k, x)=f_{l}(0, x)+o(1), & k \rightarrow 0 \text { in } \overline{\mathbf{C}^{+}} \\
f_{l}^{\prime}(k, x)=f_{l}^{\prime}(0, x)+o(1), & k \rightarrow 0 \text { in } \overline{\mathbf{C}^{+}} \\
f_{r}(k, x)=f_{r}(0, x)+o(1), & k \rightarrow 0 \text { in } \overline{\mathbf{C}^{+}} \\
f_{r}(k, x)=f_{r}^{\prime}(0, x)+o(1), & k \rightarrow 0 \text { in } \overline{\mathbf{C}^{+}}
\end{array}
$$

Hence, for example, from (1.8) and (1.9) one would expect to obtain only

$$
\frac{f_{l}^{\prime}(k, x)}{f_{l}(k, x)}=\frac{f_{l}^{\prime}(0, x)}{f_{l}(0, x)}+o(1), \quad k \rightarrow 0 \text { in } \overline{\mathbf{C}^{+}}
$$

resulting in the continuity of $f_{l}^{\prime}(\cdot, x) / f_{l}(\cdot, x)$ in $k$ at $k=0$. Thus, as compared to (1.10) the result in Theorem 2.3 is remarkable and unexpected in the sense that it establishes not only the continuity but also the differentiability of $f_{l}^{\prime}(\cdot, x) / f_{l}(\cdot, x)$ with respect to $k$ at $k=0$. This result is remarkable because it holds for any potential $V \in L_{1}^{1}(\mathbf{R})$ despite the fact that there exist potentials in this class for which $f_{l}(\cdot, x)$ and $f_{r}(\cdot, x)$ are not differentiable 
in $k$ at $k=0$ for certain values of $x$. In particular, as shown in Corollary 3.3 of [Kl88a], for an arbitrary potential in $L_{1}^{1}(\mathbf{R}), f_{l}\left(k, x_{0}\right)$ is differentiable in $k$ at $k=0$ if and only if $f_{l}\left(0, x_{0}\right)=0$. If $V(x)=V_{0} x^{-2-\epsilon}+o\left(x^{-2-\epsilon}\right)$ as $x \rightarrow+\infty$ with $V_{0} \neq 0$ and $\epsilon \in(0,1)$, as Theorem 3.1 of [Kl88b] indicates, we have $f_{l}\left(k, x_{0}\right)=f_{l}\left(0, x_{0}\right)+c|k|^{\epsilon}+o\left(|k|^{\epsilon}\right)$ as $k \rightarrow 0$ in $\overline{\mathbf{C}^{+}}$, where $c$ is nonzero and can be computed explicitly; thus $f_{l}\left(k, x_{0}\right)$ is not differentiable in $k$ at $k=0$.

\section{SMALL-ENERGY ASYMPTOTICS FOR POTENTIALS IN $L_{1}^{1}$}

For any fixed $a \in \mathbf{R}$, let $s(k, x)$ and $v(k, x)$ denote the solutions of (1.1) satisfying

$$
s(k, a)=1, \quad s^{\prime}(k, a)=0 ; \quad v(k, a)=0, \quad v^{\prime}(k, a)=1 .
$$

In fact, these solutions can be obtained from the integral equations

$$
\begin{aligned}
& s(k, x)=\cos k(x-a)+\frac{1}{k} \int_{a}^{x} d y \sin k(x-y) V(y) s(k, y), \\
& v(k, x)=\frac{\sin k(x-a)}{k}+\frac{1}{k} \int_{a}^{x} d y \sin k(x-y) V(y) v(k, y) .
\end{aligned}
$$

For each fixed $x \in \mathbf{R}$, both $s(k, x)$ and $v(k, x)$ are entire in $k$ and hence they are 'regular' solutions of (1.1).

Let us define two other regular solutions, $\phi_{l}(k, x)$ and $\phi_{r}(k, x)$, of (1.1) using linear combinations of $s(k, x)$ and $v(k, x)$ as follows:

$$
\begin{aligned}
& \phi_{l}(k, x):=f_{l}(0, a) s(k, x)+f_{l}^{\prime}(0, a) v(k, x), \\
& \phi_{r}(k, x):=f_{r}(0, a) s(k, x)+f_{r}^{\prime}(0, a) v(k, x),
\end{aligned}
$$

From (2.2)-(2.4) we obtain

$$
\phi_{l}(k, x)=f_{l}(0, a) \cos k(x-a)+f_{l}^{\prime}(0, a) \frac{\sin k(x-a)}{k}+\int_{a}^{x} d y \frac{\sin k(x-y)}{k} V(y) \phi_{l}(k, y),
$$




$$
\phi_{l}(0, x)=f_{l}(0, a)+f_{l}^{\prime}(0, a)(x-a)+\int_{a}^{x} d y(x-y) V(y) \phi_{l}(0, y)
$$

From (1.3) we get

$$
f_{l}(0, x)=1+\int_{x}^{\infty} d y(y-x) V(y) f_{l}(0, y)
$$

Note that $\phi_{l}(0, x)=f_{l}(0, x)$ for $x \in \mathbf{R}$ because both functions are solutions of (1.1) with $k=0$ and they both satisfy the same initial conditions at $x=a$. Thus, $\phi_{l}(0, x)$ is bounded for $x \geq a$; moreover using (1.2) at $k=0$ we have

$$
\phi_{l}(0, x)=1+o(1), \quad \phi_{l}^{\prime}(0, x)=o(1), \quad x \rightarrow+\infty .
$$

Letting $x \rightarrow+\infty$ in $(2.7)$, with the help of (2.9), we obtain

$$
\begin{gathered}
1=f_{l}(0, a)-a f_{l}^{\prime}(0, a)-\int_{a}^{\infty} d y y V(y) \phi_{l}(0, y) \\
0=f_{l}^{\prime}(0, a)+\int_{a}^{\infty} d y V(y) \phi_{l}(0, y) .
\end{gathered}
$$

Let us use $C$ to denote a positive constant that does not necessarily assume the same value at different appearances.

Proposition 2.1 Assume $V$ is real valued and belongs to $L_{1}^{1}(\mathbf{R})$, and fix $a \in \mathbf{R}$. Then, the solutions $\phi_{l}$ and $\phi_{r}$ defined in (2.4) and (2.5), respectively, satisfy

$$
\begin{aligned}
& \left|\phi_{l}(k, x)-\phi_{l}(0, x)\right| \leq C\left(\frac{|k(x-a)|}{1+|k(x-a)|}\right)^{2}, \quad x \geq a, \quad k \in \mathbf{R}, \\
& \left|\phi_{r}(k, x)-\phi_{r}(0, x)\right| \leq C\left(\frac{|k(x-a)|}{1+|k(x-a)|}\right)^{2}, \quad x \leq a, \quad k \in \mathbf{R} .
\end{aligned}
$$

PROOF: The proof is obtained by adapting the proof of Lemma 2.2 in [Kl88a].

Let us define

$$
P_{l}(k, a):=f_{l}^{\prime}(0, a) f_{l}(k, a)-f_{l}(0, a) f_{l}^{\prime}(k, a), \quad k \in \overline{\mathbf{C}^{+}}
$$




$$
P_{r}(k, a):=f_{r}^{\prime}(0, a) f_{r}(k, a)-f_{r}(0, a) f_{r}^{\prime}(k, a), \quad k \in \overline{\mathbf{C}^{+}} .
$$

Theorem 2.2 Assume $V$ is real valued and belongs to $L_{1}^{1}(\mathbf{R})$. Then, for each fixed $a \in \mathbf{R}$, we have $P_{l}(k, a)=-i k+o(k)$ and $P_{r}(k, a)=i k+o(k)$ as $k \rightarrow 0$ in $\overline{\mathbf{C}^{+}}$.

PROOF: Recall that the Wronskian of any two solutions of (1.1) is independent of $x$. Thus, using (2.1) we get

$$
f_{l}^{\prime}(k, a)=\left[s(k, x) ; f_{l}(k, x)\right], \quad f_{l}(k, a)=\left[f_{l}(k, x) ; v(k, x)\right],
$$

and using (2.4), (2.13), and (2.15) we have

$$
P_{l}(k, a)=\left[f_{l}(k, x) ; \phi_{l}(k, x)\right], \quad k \in \overline{\mathbf{C}^{+}} .
$$

Similarly, with the help of (2.1), (2.5), and (2.14) we obtain

$$
P_{r}(k, a)=\left[f_{r}(k, x) ; \phi_{r}(k, x)\right], \quad k \in \overline{\mathbf{C}^{+}} .
$$

Evaluating the Wronskians in (2.15) as $x \rightarrow+\infty$, and using (2.2) and (2.3), we get

$$
\begin{gathered}
f_{l}^{\prime}(k, a)=i k e^{i k a}-\int_{a}^{\infty} d y e^{i k y} V(y) s(k, y), \\
f_{l}(k, a)=e^{i k a}+\int_{a}^{\infty} d y e^{i k y} V(y) v(k, y) .
\end{gathered}
$$

From (2.4), (2.13), (2.16), and (2.17) we obtain

$$
P_{l}(k, a)=e^{i k a} f_{l}^{\prime}(0, a)-i k e^{i k a} f_{l}(0, a)+\int_{a}^{\infty} d y e^{i k y} V(y) \phi_{l}(k, y) .
$$

Using (2.10) and (2.11) we can write (2.18) as

$$
P_{l}(k, a)=-i k+J_{1}(k)+J_{2}(k)-i k\left[e^{i k a}-1\right] f_{l}(0, a)+\left[e^{i k a}-1-i k a\right] f_{l}^{\prime}(0, a)
$$

where

$$
J_{1}(k):=\int_{a}^{\infty} d y\left[e^{i k y}-1-i k y\right] V(y) \phi_{l}(0, y)
$$




$$
J_{2}(k):=\int_{a}^{\infty} d y e^{i k y} V(y)\left[\phi_{l}(k, y)-\phi_{l}(0, y)\right] .
$$

Note that $e^{i k a}-1-i k a=O\left(k^{2}\right)$ and $k\left[e^{i k a}-1\right]=O\left(k^{2}\right)$ as $k \rightarrow 0$ in $\mathbf{R}$. Using the boundedness of $\phi_{l}(0, \cdot)$ on $[a,+\infty)$ and the inequality

$$
\left|e^{i z}-i z-1\right| \leq \frac{C z^{2}}{1+z}, \quad z \geq 0,
$$

from $(2.20)$ we get

$$
\left|J_{1}(k)\right| \leq C|k| \int_{a}^{\infty} d y \frac{|k(y-a)|}{1+|k(y-a)|}(y-a)|V(y)|,
$$

and thus $J_{1}(k)=o(k)$ as $k \rightarrow 0$. Similarly, using (2.12) in (2.21) we obtain

$$
\left|J_{2}(k)\right| \leq C|k| \int_{a}^{\infty} d y \frac{|k(y-a)|}{1+|k(y-a)|}(y-a)|V(y)|,
$$

and hence $J_{2}(k)=o(k)$ as $k \rightarrow 0$. Thus, the theorem is proved when $k \rightarrow 0$ in $\mathbf{R}$. With the help of a Phragmén-Lindelöf theorem it follows that the limit is valid also when $k \rightarrow 0$ in $\overline{\mathbf{C}^{+}}$.

Our main result is contained in the following theorem.

Theorem 2.3 Assume $V$ is real valued and belongs to $L_{1}^{1}(\mathbf{R})$. For any fixed $x \in \mathbf{R}$, the Jost solutions satisfy the following:

(i) If $f_{l}(0, x) \neq 0$, then

$$
\frac{f_{l}^{\prime}(k, x)}{f_{l}(k, x)}=\frac{f_{l}^{\prime}(0, x)}{f_{l}(0, x)}+\frac{i k}{f_{l}(0, x)^{2}}+o(k), \quad k \rightarrow 0 \text { in } \overline{\mathbf{C}^{+}} .
$$

(ii) If $f_{l}^{\prime}(0, x) \neq 0$, then

$$
\frac{f_{l}(k, x)}{f_{l}^{\prime}(k, x)}=\frac{f_{l}(0, x)}{f_{l}^{\prime}(0, x)}-\frac{i k}{f_{l}^{\prime}(0, x)^{2}}+o(k), \quad k \rightarrow 0 \text { in } \overline{\mathbf{C}^{+}} .
$$

(iii) If $f_{r}(0, x) \neq 0$, then

$$
\frac{f_{r}^{\prime}(k, x)}{f_{r}(k, x)}=\frac{f_{r}^{\prime}(0, x)}{f_{r}(0, x)}-\frac{i k}{f_{r}(0, x)^{2}}+o(k), \quad k \rightarrow 0 \text { in } \overline{\mathbf{C}^{+}} .
$$


(iv) If $f_{r}^{\prime}(0, x) \neq 0$, then

$$
\frac{f_{r}(k, x)}{f_{r}^{\prime}(k, x)}=\frac{f_{r}(0, x)}{f_{r}^{\prime}(0, x)}+\frac{i k}{f_{r}^{\prime}(0, x)^{2}}+o(k), \quad k \rightarrow 0 \text { in } \overline{\mathbf{C}^{+}} .
$$

PROOF: Replacing $a$ by $x$ in (2.13), dividing both sides by $f_{l}(0, x) f_{l}(k, x)$, and using (1.8) and Theorem 2.2, we obtain (2.24). The proof of (2.25)-(2.27) is obtained in a similar manner.

Note that as the result of Theorem 2.3 is a direct consequence of Theorem 2.2, the converse is also true. Using (2.13) we can write (2.24) as

$$
-\frac{P_{l}(k, x)}{f_{l}(0, x) f_{l}(k, x)}=\frac{i k}{f_{l}(0, x)^{2}}+o(k), \quad k \rightarrow 0 \text { in } \overline{\mathbf{C}^{+}}
$$

Now from (1.8) and (2.28) we get $P_{l}(k, x)=-i k+o(k)$ as $k \rightarrow 0$ in $\overline{\mathbf{C}^{+}}$. Similarly, $P_{r}(k, x)=i k+o(k)$ is a direct consequence of (2.26) and (2.27). An independent proof of Theorem 2.3 is given in the Appendix based on the solutions of a Riccati equation. For an analog of Theorem 2.3 for the radial Schrödinger equation, the reader is referred to [Ak00].

\section{APPLiCATIONS TO POTENTIALS IN $L_{1}^{1}$}

Theorem 2.3 can be used to readily evaluate the small- $k$ asymptotics of the scattering coefficients. We can write (1.4) in the form

$$
\frac{2 i k}{T(k)}=f_{r}(k, x) f_{l}(k, x)\left[\frac{f_{l}^{\prime}(k, x)}{f_{l}(k, x)}-\frac{f_{r}^{\prime}(k, x)}{f_{r}(k, x)}\right] .
$$

For example, in the exceptional case, using (1.7), (2.24), and (2.26) in (3.1), we get

$$
\frac{2 i k}{T(k)}=i k\left[\frac{f_{r}(0, x)}{f_{l}(0, x)}+\frac{f_{l}(0, x)}{f_{r}(0, x)}\right]+o(k), \quad k \rightarrow 0 \text { in } \overline{\mathbf{C}^{+}}
$$

which gives us

$$
T(k)=\frac{2 \gamma}{\gamma^{2}+1}+o(1), \quad k \rightarrow 0 \text { in } \overline{\mathbf{C}^{+}}
$$


where $\gamma$ is the constant defined in (1.7). Similarly, writing (1.5) as

$$
\frac{2 i k R(k)}{T(k)}=f_{r}(k, x) f_{l}(-k, x)\left[\frac{f_{r}^{\prime}(k, x)}{f_{r}(k, x)}-\frac{f_{l}^{\prime}(-k, x)}{f_{l}(-k, x)}\right],
$$

and using (1.7), (2.24), and (2.26) in (3.3), in the exceptional case we get

$$
\frac{2 i k R(k)}{T(k)}=i k\left[\frac{f_{r}(0, x)}{f_{l}(0, x)}-\frac{f_{l}(0, x)}{f_{r}(0, x)}\right]+o(k), \quad k \rightarrow 0 \text { in } \mathbf{R},
$$

which, with the help of (3.2), gives us

$$
R(k)=\frac{1-\gamma^{2}}{\gamma^{2}+1}+o(1), \quad k \rightarrow 0 \text { in } \mathbf{R} .
$$

In a similar manner, (1.7), (2.24), and (2.26) can be used in (1.6) in the exceptional case in order to obtain

$$
L(k)=\frac{\gamma^{2}-1}{\gamma^{2}+1}+o(1), \quad k \rightarrow 0 \text { in } \mathbf{R}
$$

Next, let us explore the connection between Theorem 2.3 and the potentials with supports contained in a half line. If $V$ vanishes for $x<0$, we have

$$
f_{l}(k, x)=\frac{e^{i k x}}{T(k)}+\frac{e^{-i k x} L(k)}{T(k)}, \quad x \leq 0,
$$

and if $V$ vanishes for $x>0$, we have

$$
f_{r}(k, x)=\frac{e^{-i k x}}{T(k)}+\frac{e^{i k x} R(k)}{T(k)}, \quad x \geq 0 .
$$

Theorem 3.1 If $V$ is real valued, belongs to $L_{1}^{1}(\mathbf{R})$, and vanishes for $x<0$, then as $k \rightarrow 0$ in $\overline{\mathbf{C}^{+}}$the corresponding reflection coefficient from the left satisfies

$$
L(k)=\left\{\begin{array}{l}
-1+2 i k \frac{f_{l}(0,0)}{f_{l}^{\prime}(0,0)}+2 k^{2} \frac{1+f_{l}(0,0)^{2}}{f_{l}^{\prime}(0,0)^{2}}+o\left(k^{2}\right), \\
\frac{f_{l}(0,0)^{2}-1}{f_{l}(0,0)^{2}+1}+o(1), \quad \text { if } f_{l}^{\prime}(0,0)=0 .
\end{array}\right.
$$


Similarly, if $V$ is real valued, belongs to $L_{1}^{1}(\mathbf{R})$, and vanishes for $x>0$, then as $k \rightarrow 0$ in $\overline{\mathbf{C}^{+}}$the corresponding reflection coefficient from the right satisfies

$$
R(k)= \begin{cases}-1-2 i k \frac{f_{r}(0,0)}{f_{r}^{\prime}(0,0)}+2 k^{2} \frac{1+f_{r}(0,0)^{2}}{f_{r}^{\prime}(0,0)^{2}}+o\left(k^{2}\right), & \text { if } f_{r}^{\prime}(0,0) \neq 0, \\ \frac{f_{r}(0,0)^{2}-1}{f_{r}(0,0)^{2}+1}+o(1), & \text { if } f_{r}^{\prime}(0,0)=0 .\end{cases}
$$

We refer the reader to Theorem 8.1 of $[\mathrm{Ak} 99]$ for a proof of $(3.8)$ when $f_{l}^{\prime}(0,0) \neq 0$; the case $f_{l}^{\prime}(0,0)=0$ follows from (3.5) and (3.6) using $\gamma=f_{l}(0,0)$. Similarly, the proof of $(3.9)$ when $f_{r}^{\prime}(0,0) \neq 0$ was given in Corollary 8.2 of $[\mathrm{Ak} 99]$, and the case $f_{r}^{\prime}(0,0)=0$ follows from (3.4) and (3.7) using $\gamma=1 / f_{r}(0,0)$. Next, we show that Theorem 2.3 and Theorem 3.1 are closely related to each other.

Theorem 3.2 The result of Theorem 3.1 can be directly derived from that of Theorem 2.3 and vice versa.

PROOF: If $V$ vanishes for $x<0$, from (3.6) we get

$$
L(k)=\frac{-1+i k f_{l}(k, 0) / f_{l}^{\prime}(k, 0)}{1+i k f_{l}(k, 0) / f_{l}^{\prime}(k, 0)}
$$

If $V$ is generic, then $f_{l}^{\prime}(0,0) \neq 0$, and hence using (2.25) in (3.10) we obtain the first case in (3.8). If $f_{l}^{\prime}(0,0)=0$, then we have (3.5), and hence the second case in (3.8) holds.

In a similar manner, if $V$ vanishes for $x>0$, then from (3.7) we get

$$
R(k)=\frac{1+i k f_{r}(k, 0) / f_{r}^{\prime}(k, 0)}{-1+i k f_{r}(k, 0) / f_{r}^{\prime}(k, 0)} .
$$

If $V$ is generic, then $f_{r}^{\prime}(0,0) \neq 0$, and hence using (2.27) in (3.11) we obtain the first case in (3.9). If $f_{r}^{\prime}(0,0)=0$, then we have (3.4), and thus the second case in (3.9) holds.

In order to show that (3.8) of Theorem 3.1 implies (2.24) and (2.25) of Theorem 2.3, let us choose

$$
V_{2}(x):=\left\{\begin{array}{l}
V(x), \quad x \geq \max \{0, a\}, \\
0, \quad x<\max \{0, a\},
\end{array}\right.
$$


so that $V_{2}$ vanishes when $x<0$. Denoting the corresponding Jost solution from the left by $f_{l 2}(k, x)$, we have

$$
f_{l}(k, a)=f_{l 2}(k, a), \quad f_{l}^{\prime}(k, a)=f_{l 2}^{\prime}(k, a), \quad k \in \overline{\mathbf{C}^{+}}
$$

$$
f_{l 2}(0, a)=f_{l 2}(0,0)+a f_{l 2}^{\prime}(0,0)
$$

Using (3.6) we get

$$
\frac{f_{l 2}^{\prime}(k, a)}{f_{l 2}(k, a)}=i k \frac{e^{2 i k a}-L_{2}(k)}{e^{2 i k a}+L_{2}(k)} .
$$

As $k \rightarrow 0$ in $\overline{\mathbf{C}^{+}}$, using $(3.8)$ we get

$$
\begin{aligned}
& i k \frac{e^{2 i k a}-L_{2}(k)}{e^{2 i k a}+L_{2}(k)} \\
& \quad=\left\{\begin{array}{l}
\frac{f_{l 2}^{\prime}(0,0)}{f_{l 2}(0,0)+a f_{l 2}^{\prime}(0,0)}+\frac{i k}{\left[f_{l 2}(0,0)+a f_{l 2}^{\prime}(0,0)\right]^{2}}+o(k), \quad f_{l 2}^{\prime}(0,0) \neq 0, \\
\frac{i k}{f_{l 2}(0,0)^{2}}+o(k), \quad f_{l 2}^{\prime}(0,0)=0 .
\end{array}\right.
\end{aligned}
$$

From (3.14)-(3.16) we obtain

$$
\frac{f_{l 2}^{\prime}(k, a)}{f_{l 2}(k, a)}=\left\{\begin{array}{l}
\frac{f_{l 2}^{\prime}(0, a)}{f_{l 2}(0, a)}+\frac{i k}{f_{l 2}(0, a)^{2}}+o(k), \quad f_{l 2}^{\prime}(0, a) \neq 0 \\
\frac{i k}{f_{l 2}(0, a)^{2}}+o(k), \quad f_{l 2}^{\prime}(0, a)=0
\end{array}\right.
$$

Because of (3.12) and (3.13), (3.17) is equivalent to (2.24) and (2.25). The proof that the result in (3.9) implies those in (2.26) and (2.27) is obtained in a similar manner by choosing

$$
V_{1}(x):=\left\{\begin{array}{l}
V(x), \quad x \leq \min \{0, a\}, \\
0, \quad x>\min \{0, a\},
\end{array}\right.
$$


so that $V_{1}$ vanishes when $x>0$, and by using the fact that $f_{r}(k, a)=f_{r 1}(k, a)$ and $f_{r}^{\prime}(k, a)=f_{r 1}^{\prime}(k, a)$, where $f_{r 1}(k, x)$ denotes the Jost solution from the right corresponding to $V_{1}$.

\section{SMALL-ENERGY ASYMPTOTICS FOR POTENTIALS IN $L_{2}^{1}$}

The proofs given in Sections 2 and 3 can be modified to study the small- $k$ asymptotics when the potential is real valued and belongs to the more restrictive class $L_{2}^{1}(\mathbf{R})$.

Our first result is the analog of Theorem 2.2.

Theorem 4.1 Assume $V$ is real valued and belongs to $L_{2}^{1}(\mathbf{R})$. Then, for each fixed $a \in$ $\mathbf{R}$, the functions $P_{l}(k, a)$ and $P_{r}(k, a)$ defined in (2.13) and (2.14), respectively, satisfy $P_{l}(k, a)=-i k+O\left(k^{2}\right)$ and $P_{r}(k, a)=i k+O\left(k^{2}\right)$ as $k \rightarrow 0$ in $\overline{\mathbf{C}^{+}}$.

PROOF: The proof of Theorem 2.2 can easily be modified. From (2.19) it is clear that we only need to show that $J_{1}(k)=O\left(k^{2}\right)$ and $J_{2}(k)=O\left(k^{2}\right)$ as $k \rightarrow 0$ in $\mathbf{R}$, which follow from (2.22) and (2.23) by taking the factor $|k|$ in the numerators of the integrands outside the integrals; the resulting integrals remain finite because $\int_{a}^{\infty} d y(y-a)^{2}|V(y)|$ converges if $V \in L_{2}^{1}(\mathbf{R})$.

The next result is the analog of Theorem 2.3.

Theorem 4.2 Assume $V$ is real valued and belongs to $L_{2}^{1}(\mathbf{R})$. For any fixed $x \in \mathbf{R}$, the Jost solutions satisfy $(2.24)-(2.27)$ but with $o(k)$ replaced by $O\left(k^{2}\right)$.

PROOF: The proof is given by modifying the proof of Theorem 2.3, i.e. by showing that the results stated are implied by Theorem 4.1. When $V \in L_{2}^{1}(\mathbf{R})$, instead of (1.8) one has [DT79]

$$
f_{l}(k, x)=f_{l}(0, x)+k \dot{f}_{l}(0, x)+o(k), \quad k \rightarrow 0 \text { in } \overline{\mathbf{C}^{+}},
$$

where the overdot denotes the derivative with respect to $k$. Dividing both sides of $(2.13)$ 
by $f_{l}(0, a) f_{l}(k, a)$ and by using Theorem 4.1 , we get

$$
\frac{f_{l}^{\prime}(k, x)}{f_{l}(k, x)}=\frac{f_{l}^{\prime}(0, x)}{f_{l}(0, x)}+\frac{i k}{f_{l}(0, x)^{2}}+O\left(k^{2}\right), \quad k \rightarrow 0 \text { in } \overline{\mathbf{C}^{+}} .
$$

Similarly, with the help of

$$
f_{r}(k, x)=f_{r}(0, x)+k \dot{f}_{r}(0, x)+o(k), \quad k \rightarrow 0 \text { in } \overline{\mathbf{C}^{+}},
$$

we obtain

$$
\frac{f_{r}^{\prime}(k, x)}{f_{r}(k, x)}=\frac{f_{r}^{\prime}(0, x)}{f_{r}(0, x)}-\frac{i k}{f_{r}(0, x)^{2}}+O\left(k^{2}\right), \quad k \rightarrow 0 \text { in } \overline{\mathbf{C}^{+}} .
$$

The analogs of (2.25) and (2.27) are obtained similarly.

In the next theorem the small-energy asymptotics of the scattering coefficients are obtained.

Theorem 4.3 Assume $V$ is real valued and belongs to $L_{2}^{1}(\mathbf{R})$. Then, as $k \rightarrow 0$ in $\overline{\mathbf{C}^{+}}$we have

$$
T(k)=\left\{\begin{array}{l}
\frac{2 i k}{W_{0}}+\frac{2 k^{2}\left(A_{1}+A_{2}\right)}{W_{0}^{2}}+o\left(k^{2}\right), \quad \text { generic case }, \\
\frac{2 \gamma}{\gamma^{2}+1}+O(k), \quad \text { exceptional case, }
\end{array}\right.
$$

and as $k \rightarrow 0$ in $\mathbf{R}$ we have

$$
\begin{aligned}
& L(k)=\left\{\begin{array}{lr}
-1+\frac{2 i k A_{1}}{W_{0}}+o(k), & \text { generic case }, \\
\frac{\gamma^{2}-1}{\gamma^{2}+1}+O(k), & \text { exceptional case },
\end{array}\right. \\
& R(k)=\left\{\begin{array}{lr}
-1+\frac{2 i k A_{2}}{W_{0}}+o(k), & \text { generic case } \\
\frac{1-\gamma^{2}}{\gamma^{2}+1}+O(k), & \text { exceptional case }
\end{array}\right.
\end{aligned}
$$

where $\gamma$ is the constant in (1.7), $W_{0}$ is the real nonzero constant given by

$$
W_{0}:=\left[f_{r}(0, x) ; f_{l}(0, x)\right],
$$


and $A_{1}$ and $A_{2}$ are the real constants (independent of $x$ ) defined as

$$
A_{1}:=\frac{f_{l}(0, x)-i W_{0} \dot{f}_{r}(0, x)}{f_{r}(0, x)}, \quad A_{2}:=\frac{f_{r}(0, x)-i W_{0} \dot{f}_{l}(0, x)}{f_{l}(0, x)} .
$$

PROOF: The proof of (4.5) is obtained by using (4.1)-(4.4) in (3.1). The proof of (4.7) is given in a similar manner with the help of (3.3). Analogously, (4.6) is proved.

The result given in Theorem 4.3 can actually be improved further in the exceptional case; the $O(k)$-terms in (4.5)-(4.7) can be explicitly evaluated, and it can be shown that $T(k), L(k)$, and $R(k)$ are all differentiable at $k=0$ when $V \in L_{2}^{1}(\mathbf{R})$. We refer the reader to Theorem 4.10 and Example 5.1 of [AKV00] for the explicit expressions for $\dot{T}(0), \dot{L}(0)$, and $\dot{R}(0)$.

It may be surprising at a first sight that the right-hand sides in (4.8) are independent of $x$. This $x$-independence can be seen as follows. In the generic case, $f_{l}(0, x)$ and $f_{r}(0, x)$ are linearly independent solutions of (1.1) with $k=0$. Thus, any other solutions, among which are $\dot{f}_{l}(0, x)$ and $\dot{f}_{r}(0, x)$, can be expressed as linear combinations of $f_{l}(0, x)$ and $f_{r}(0, x)$, which gives us (4.8).

The following theorem is the analog of Theorem 3.1.

Theorem 4.4 Assume $V$ is real valued, belongs to $L_{2}^{1}(\mathbf{R})$, and vanishes for $x<0$; then as $k \rightarrow 0$ in $\overline{\mathbf{C}^{+}}$the corresponding reflection coefficient from the left satisfies

$$
L(k)=\left\{\begin{array}{l}
-1+2 i k \frac{f_{l}(0,0)}{f_{l}^{\prime}(0,0)}+2 k^{2} \frac{1+f_{l}(0,0)^{2}}{f_{l}^{\prime}(0,0)^{2}}+O\left(k^{3}\right), \\
\frac{f_{l}(0,0)^{2}-1}{f_{l}(0,0)^{2}+1}+O(k), \quad \text { if } f_{l}^{\prime}(0,0)=0 .
\end{array}\right.
$$

Similarly, assume $V$ is real valued, belongs to $L_{2}^{1}(\mathbf{R})$, and vanishes for $x>0$; then as $k \rightarrow 0$ in $\overline{\mathbf{C}^{+}}$the corresponding reflection coefficient from the right satisfies

$$
R(k)= \begin{cases}-1-2 i k \frac{f_{r}(0,0)}{f_{r}^{\prime}(0,0)}+2 k^{2} \frac{1+f_{r}(0,0)^{2}}{f_{r}^{\prime}(0,0)^{2}}+O\left(k^{3}\right), & \text { if } f_{r}^{\prime}(0,0) \neq 0, \\ \frac{f_{r}(0,0)^{2}-1}{f_{r}(0,0)^{2}+1}+O(k), & \text { if } f_{r}^{\prime}(0,0)=0 .\end{cases}
$$


PROOF: The proof is obtained as in the first two paragraphs of the proof of Theorem 3.2.

Let us remark that, by proceeding as in the proof of Theorem 3.2, it is possible to prove the result of Theorem 4.4 directly by using the result of Theorem 4.2 and vice versa.

\section{CHARACTERIZATION PROBLEM REVISITED}

In this section we discuss some implications of the results of Section 3 for the characterization problem of inverse scattering theory for real-valued potentials in $V \in L_{1}^{1}(\mathbf{R})$. By this we mean the problem of finding necessary and sufficient conditions on the scattering data which guarantee that there is exactly one real-valued potential $V \in L_{1}^{1}(\mathbf{R})$ corresponding to that data. Such characterizations were given by Melin [Me85] and Marchenko [Ma86]. It is known that one can construct $V$ uniquely from either the left scattering data $\left\{R,\left\{\kappa_{j}\right\},\left\{c_{l ; j}\right\}\right\}$ or the right scattering data $\left\{L,\left\{\kappa_{j}\right\},\left\{c_{r ; j}\right\}\right\}$. Here $\kappa_{j}$ for $j=1, \ldots, N$ are

$N$ distinct positive numbers such that $-\kappa_{j}^{2}$ represent the bound state energies for $V$, and $c_{l ; j}$ and $c_{r ; j}$ are positive constants called bound-state norming constants; $c_{l ; j}$ and $c_{r ; j}$ are the reciprocals of the norms of the eigenfunctions $f_{l}\left(i \kappa_{j}, \cdot\right)$ and $f_{r}\left(i \kappa_{j}, \cdot\right)$, respectively.

Among the characterization conditions listed in Theorem 6.1 of [Me85] and Theorem 3.5.1 of [Ma86] is the condition

$$
\lim _{k \rightarrow 0} \frac{k}{T(k)}[R(k)+1]=0, \quad k \in \mathbf{R},
$$

and it plays an important role in the reconstruction of $V$ from the scattering data. Condition (5.1) provides a way of proving the characterization theorem without using the continuity of $R(k)$ and $T(k)$ at $k=0$, which was not known at the time (cf. p. 303 of [Ma86]). For $V \in L_{1}^{1}(\mathbf{R})$, the continuity of the scattering coefficients at $k=0$ was later proved in [K188a]. Since the continuity of $R$ for $k \in \mathbf{R} \backslash\{0\}$ is already listed in both [Me85] and [Ma86] among the characterization conditions, and since we now know that $R(k)$ is continuous at $k=0$, it is worth asking whether condition (5.1) can simply be omitted and 
replaced by the condition that $R(k)$ be continuous at $k=0$. As our next theorem shows, this is indeed the case.

Recall the formulas [Fa64,DT79,CS89]

$$
\begin{aligned}
& T(k)=\left\{\begin{array}{l}
\left(\prod_{j=1}^{N} \frac{k+i \kappa_{j}}{k-i \kappa_{j}}\right) \exp \left(\frac{1}{2 \pi i} \int_{-\infty}^{\infty} d t \frac{\log \left(1-|R(t)|^{2}\right)}{t-k}\right), \quad k \in \mathbf{C}^{+}, \\
\lim _{\epsilon \rightarrow 0^{+}} T(k+i \epsilon), \quad k \in \mathbf{R},
\end{array}\right. \\
& L(k)=-\frac{R(k)^{*} T(k)}{T(k)^{*}}, \quad k \in \mathbf{R},
\end{aligned}
$$

where the asterisk denotes complex conjugation, and define

$$
\hat{R}(\alpha):=\frac{1}{2 \pi} \int_{-\infty}^{\infty} d k R(k) e^{i k \alpha}, \quad \hat{L}(\alpha):=\frac{1}{2 \pi} \int_{-\infty}^{\infty} d k L(k) e^{i k \alpha}
$$

We propose the following simplified list of necessary and sufficient conditions as a characterization of real-valued potentials in $L_{1}^{1}(\mathbf{R})$.

Theorem 5.1 In order for the data $\left\{R,\left\{\kappa_{j}\right\},\left\{c_{l ; j}\right\}\right\}$ to be the left scattering data for (1.1) with a real-valued potential $V \in L_{1}^{1}(\mathbf{R})$, it is necessary and sufficient that the following conditions hold:

(i) $R(-k)=R(k)^{*}$ for $k \in \mathbf{R}$.

(ii) $R(k)$ is continuous for $k \in \mathbf{R}, R(0) \in[-1,1),|R(k)| \leq 1-C k^{2}\left(1+k^{2}\right)^{-1}$ for some constant $C>0$, and $R(k)=o(1 / k)$ as $k \rightarrow \pm \infty$.

(iii) The function $k / T(k)$, where $T(k)$ is given by $(5.2)$, is continuous in $\overline{\mathbf{C}^{+}}$.

(iv) The functions $\hat{R}$ and $\hat{L}$ defined in (5.4), where $L(k)$ is obtained from (5.3), are absolutely continuous. Moreover, $\hat{R}^{\prime} \in L_{1}^{1}(a,+\infty)$ and $\hat{L}^{\prime} \in L_{1}^{1}(-\infty, a)$ for every $a \in \mathbf{R}$.

PROOF: It suffices to show that (5.1) is a consequence of (ii) and (iii). To see this, we argue as follows. If $R(0)=-1$, then $|T(k)|^{2}=1-|R(k)|^{2} \geq C k^{2}\left(1+k^{2}\right)^{-1}$. Note that 
the relation $|T(k)|^{2}=1-|R(k)|^{2}$ for $k \neq 0$ follows from (5.2). Hence $|k / T(k)| \leq \sqrt{2 / C}$ for $k \in(-1,1)$, even when $k=0$ because, by (iii), $k / T(k)$ has a limit as $k \rightarrow 0$. Hence (5.1) follows from the continuity of $R$ at $k=0$. If $R(0) \in(-1,1)$, then there is a positive constant $c_{0}$ such that $|T(k)| \geq c_{0}$ for $k \in(-1,1) \backslash\{0\}$ because $|T(k)|^{2}=1-|R(k)|^{2}$ and $R(k)$ is continuous at $k=0$. Hence $k / T(k) \rightarrow 0$ as $k \rightarrow 0$ and (5.1) follows.

Note that in (ii) of Theorem 5.1 we have incorporated the continuity of $R$ at $k=0$, together with the condition that $R(0) \in[-1,1)$, which is also necessary in view of (3.4) and the fact that $R(0)=-1$ in the generic case. The other conditions are essentially the same as those in [Ma86].

\section{APPENDIX: A SECOND PROOF OF THEOREM 2.3}

PROOF: We will prove only (2.24) because the proof for (2.25)-(2.27) is similar. There is no loss of generality in giving the proof of (2.24) at $x=0$ only, and hence we assume $f_{l}(0,0) \neq 0$. It is enough to give the proof for real $k$ because, as in the proof of Theorem 2.2, the result can be extended to $k \in \overline{\mathbf{C}^{+}}$with the help of a Phragmén-Lindelöf theorem. Furthermore, it is sufficient to assume $k>0$ because replacing a real $k$ by $-k$ amounts to taking complex conjugation in all the terms with which we will deal.

The logarithmic spatial derivatives of the solutions of (1.1) satisfy the Riccati equation

$$
\eta^{\prime}(k, x)+\eta(k, x)^{2}+k^{2}=V(x), \quad x \in \mathbf{R} .
$$

Let us define $\eta_{l}(k, x):=f_{l}^{\prime}(k, x) / f_{l}(k, x)$. Then, $\eta_{l}$ is a solution of (A.1) satisfying the boundary condition

$$
\eta_{l}(k, x)=i k+o(1), \quad x \rightarrow+\infty .
$$

From (1.3) we infer that

$$
\eta_{l}(k, x)=i k+O\left(\int_{x}^{\infty} d y|V(y)|\right)=i k+o(1 / x), \quad x \rightarrow+\infty .
$$


Let $h(k, x):=\eta_{l}(k, x)-i k$ and note that $h(k, x)$ obeys the Riccati equation

$$
h^{\prime}(k, x)+2 i k h(k, x)=V(x)-h(k, x)^{2}, \quad x \in \mathbf{R} .
$$

We solve (A.2) by iteration so that $h(k, x)=\lim _{n \rightarrow+\infty} h_{n}(k, x)$, where $h_{0}(k, x)=0$ and

$$
h_{n}(k, x)=e^{-2 i k x} \int_{x}^{\infty} d y\left[-V(y)+h_{n-1}(k, y)^{2}\right] e^{2 i k y}, \quad n \geq 1
$$

We first construct the solution $h(k, x)$ on the $x$-interval $[\rho,+\infty)$ with $\rho>0$ and so large that

$$
\int_{\rho}^{\infty} d y y|V(y)|<\frac{1}{4}
$$

On this interval we have the estimate

$$
\left|h_{n}(k, x)\right| \leq 2 \int_{x}^{\infty} d y|V(y)|, \quad x \geq \rho, \quad n \geq 0
$$

Obviously, (A.5) holds for $n=0$; assuming it is true for $h_{n}(k, x)$ we conclude using (A.3) and (A.4) that

$$
\begin{aligned}
\left|h_{n+1}(k, x)\right| & \leq \int_{x}^{\infty} d y|V(y)|+4 \int_{x}^{\infty} d y\left(\int_{y}^{\infty} d s|V(s)|\right)^{2} \\
& \leq\left(\int_{x}^{\infty} d y|V(y)|\right)\left[1+4 \int_{x}^{\infty} d y y|V(y)|\right] \\
& \leq 2 \int_{x}^{\infty} d y|V(y)|
\end{aligned}
$$

where during the computation we have dropped a nonpositive term because $x \geq 0$. Note also that (A.4) guarantees that $f_{l}(0, x)>0$ when $x \geq \rho$, which can be seen with the help of (2.8). Using (A.5) and arguing by induction we get

$$
\left|h_{n+1}(k, x)-h_{n}(k, x)\right| \leq 2\left(4 \int_{x}^{\infty} d y y|V(y)|\right)^{n} \int_{x}^{\infty} d t|V(t)|, \quad x \geq \rho, \quad n \geq 0 .
$$

Thus (A.4) guarantees that the sequence $\left\{h_{n}(k, x)\right\}$ converges, uniformly in $x$ for $x \geq \rho$ and uniformly in $k$ for $k \geq 0$. From the integral equation for $h(k, x)$ obtained by letting 
$n \rightarrow+\infty$ in (A.3) we infer that

$$
\begin{aligned}
h(k, x)-h(0, x)= & \int_{x}^{\infty} d y\left[-V(y)+h(k, y)^{2}\right]\left[e^{2 i k(y-x)}-1\right] \\
& +\int_{x}^{\infty} d y\left[h(k, y)^{2}-h(0, y)^{2}\right] .
\end{aligned}
$$

Hence, using (A.4), (A.5) when $n \rightarrow+\infty$, and the estimate

$$
\int_{x}^{\infty} d y y\left(\int_{y}^{\infty} d s|V(s)|\right)^{2} \leq\left(\int_{x}^{\infty} d y y|V(y)|\right)^{2},
$$

we obtain

$$
\begin{aligned}
|h(k, x)-h(0, x)| \leq & 2 k\left[\int_{x}^{\infty} d y y|V(y)|+4 \int_{x}^{\infty} d y y\left(\int_{y}^{\infty} d s|V(s)|\right)^{2}\right] \\
& +4 \int_{x}^{\infty} d y\left(\int_{y}^{\infty} d s|V(s)|\right)|h(k, y)-h(0, y)| \\
\leq & k+4 \int_{x}^{\infty} d y\left(\int_{y}^{\infty} d s|V(s)|\right)|h(k, y)-h(0, y)| .
\end{aligned}
$$

Applying Gronwall's lemma to (A.7) we get

$$
|h(k, x)-h(0, x)| \leq k e^{4 \int_{x}^{\infty} d y y|V(y)|} .
$$

Then, for $x \geq \rho$, the existence of $\dot{h}(0, x)$ and hence that of $\dot{\eta}_{l}(0, x)$ follow from (A.6), (A.8), and the Lebesgue dominated convergence theorem.

When $x<\rho$ we proceed as follows. Differentiating (A.1) with respect to $k$, multiplying the resulting equation on both sides by $f_{l}(k, x)$, and integrating over $(x, \rho)$, we get

$$
\dot{\eta}(k, x)=f_{l}(k, \rho)^{2} f_{l}(k, x)^{-2} \dot{\eta}(k, \rho)+2 k f_{l}(k, x)^{-2} \int_{x}^{\rho} d y f_{l}(k, y)^{2},
$$

where $k \neq 0$. Note that, as stated in Section 1 , we have $f_{l}(k, x) \neq 0$ when $k \in \mathbf{R} \backslash\{0\}$. Since we assume $f_{l}(0, x) \neq 0$, we can let $k \rightarrow 0$ in (A.9) so that

$$
\dot{\eta}_{l}(0, x)=\frac{f_{l}(0, \rho)^{2}}{f_{l}(0, x)^{2}} \dot{\eta}_{l}(0, \rho)
$$


Letting $\rho \rightarrow+\infty$ in (A.10), and using $f_{l}(0, \rho) \rightarrow 1$ and $\dot{\eta}(0, \rho) \rightarrow i$, we obtain $\dot{\eta}(0,0)=$ $i / f_{l}(0,0)^{2}$. This proves $(2.24)$.

Acknowledgment. The research leading to this article was supported in part by the National Science Foundation under grant DMS-9803219.

\section{REFERENCES}

[Ak99] T. Aktosun, On the Schrödinger equation with steplike potentials, J. Math. Phys. 40, 5289-5305 (1999).

[Ak00] T. Aktosun, Factorization and small-energy asymptotics for the radial Schrödinger equation, J. Math. Phys. 41, 4262-4270 (2000).

[AKV00] T. Aktosun, M. Klaus, and Cornelis van der Mee, Small-energy asymptotics of the scattering matrix for the matrix Schrödinger equation on the line, IMA Preprint \#1716, August 2000 [http://www.ima.umn.edu/preprints/aug2000/aug2000.html].

[CS89] K. Chadan and P. C. Sabatier, Inverse problems in quantum scattering theory, 2nd ed., Springer, New York, 1989.

[DT79] P. Deift and E. Trubowitz, Inverse scattering on the line, Comm. Pure Appl. Math. 32, 121-251 (1979).

[Fa64] L. D. Faddeev, Properties of the S-matrix of the one-dimensional Schrödinger equation, Am. Math. Soc. Transl. (Ser. 2) 65, 139-166 (1964) [Trudy Mat. Inst. Steklova 73, 314-336 (1964) (Russian)].

[Kl88a] M. Klaus, Low-energy behaviour of the scattering matrix for the Schrödinger equation on the line, Inverse Problems 4, 505-512 (1988).

[Kl88b] M. Klaus, Exact behavior of Jost functions at low energy, J. Math. Phys. 29, 148-154 (1988). 
[Ma86] V. A. Marchenko, Sturm-Liouville operators and applications, Birkhäuser, Basel, 1986.

[Me85] A. Melin, Operator methods for inverse scattering on the real line, Comm. Partial Differential Equations 10, 677-766 (1985). 